\title{
Passage des Gravilliers, Paris, 1er juin 2009: entre champ visuel et territoire sonore
}

\author{
GHISLAINE PERICHET
}

Vidéaste, commissaire d'exposition associée à l'équipe de la Galerie Michel Journiac (UFR04 d'Arts Plastiques et Sciences de l'Art - Université Paris 1- Panthéon-Sorbonne ), Ghislaine Perichet mène une réflexion autour du point de vue. Ses images et scénographies d'images vidéo s'expérimentent à travers les dispositifs d'installation audiovisuels qui interrogent la place du visiteur, sa présence sollicitée entre champ visuel et territoire sonore. 


\section{- RÉSUMÉ}

À défaut d'enquêter sur le lieu dans la perspective d'y trouver l'indice qui détourne l'attention, plus que chercher d'autres espaces, c'est au gré de mes déambulations que je tente un " être-là ", une présence au lieu, à l'espace que je traverse en même temps qu'il me parle et me touche. Au passage des Gravilliers, placé au centre du dispositif d'enregistrement de l'image panoramique autour duquel s'enroulait l'architecture et déambulaient les personnages, mon corps s'avérait l'élément catalyseur à l'origine de la représentation. Quelle place aujourd'hui réserver au visiteur qui expérimente Passage des Gravilliers, le dispositif de mise en scène de l'image en mouvement imaginé pour tenter de restituer le temps d'une expérience, l'histoire à même de s'inventer, entre champ visuel et territoire sonore?

\section{- MOTS-CLÉS}

Expérience, panoramique, entre-deux, champ visuel, territoire sonore, performance, dispositif de mise en scène.

\section{- RESUMO}

Evito investigar o lugar com o objetivo de encontrar o índice que desvia a atenção, mais do que buscar outros espaços, é isto que procuro com minhas andanças. Busco um "estar lá". A presença de um lugar que eu cruzo ao mesmo tempo em que ele me atravessa. A travessa Passage des Gravilliers está colocada no centro do dispositivo de gravação da imagem panorâmica que se desenrola em torno da arquitetura e do passear dos personagens, meu corpo é o catalisador por trás do espetáculo. Que lugar se reserva hoje para o visitante experimenta a obra Passage des Gravilliers, dispositivo encenado de imagem em movimento, concebido para tentar restaurar o tempo de uma experiência, a própria história para inventar, entre o campo visual e o território sonoro?

\section{- PALAVRAS-CHAVE}

Experiência, panorâmica, campo visual, território sonoro, performance, dispositivos de encenação.

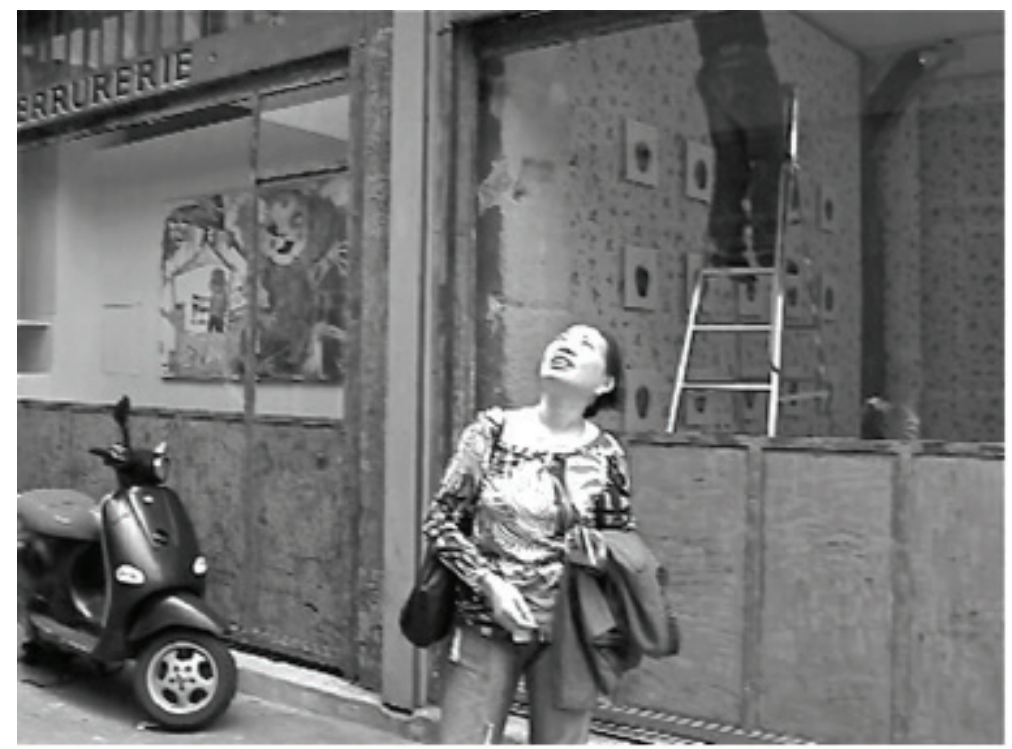

Figure 1 - Passage des Gravilliers, Paris, 1er juin 2009 (capture d'écran). Installation DVD 1 écran, projection vidéo en boucle, 5'37', couleur, son. 


\section{Passage des Gravilliers}

Passage des Gravilliers : une vidéo, un plan-séquence et travelling latéral de presque cinq minutes enregistré à Paris, le 1er juin 2009 au passage des Gravilliers. Aujourd'hui, une image panoramique projetée en boucle à l'intérieur d'un dispositif architectural spécifique visant à retracer l'expérience vécue in situ.

La scène s'ouvre au moment où une voix féminine retentit, le moment où la femme entre dans le champ de la caméra pendant que la voix de l'homme resté dans l'ombre continue de faire écho à nos présences découpées sur fond de paysage urbain archistructuré, le contexte architecturé d'où la femme s'extrait en franchissant la porte cochère, le moment où prend fin la scène. Au temps des relations hier imprévues s'accorde aujourd'hui le temps de la scénographie des images, le dispositif de la mise en scène impromptue.

Le regard panoramique ouvre la perception au champ de la représentation, il est la trame à travers laquelle les relations dont il initie le mouvement prennent forme, il est le lieu où se cristallise la rencontre, le temps de la performance improvisée. Là où je vois et suis vue, là où mon corps pris dans la dynamique des interférences se met en mouvement, entre présences réelles et présences réfléchies sur les vitrines, entre territoire visuel et environnement sonore, j'entrais dans la danse. La femme que je suivais du regard, celle que j'entendais en même temps qu'elle me voyait, traversant le champ de la caméra ou bien s'en extrayant sous l'œil inquisiteur de la caméra ; entre elles, l'actrice en quête de l'interlocuteur providentiel et la vidéaste, figure d'attraction à la conquête d'une rencontre improbable autour de laquelle se nouaient les relations, quelque chose passa. Quelque chose se passa, de l'ordre de l'émotion à l'origine de la fiction. Les présences s'accordèrent un temps, le temps de la scène inédite enregistrée dans le cadre d'une unité spatio-temporelle spécifique, le temps d'une expérience.

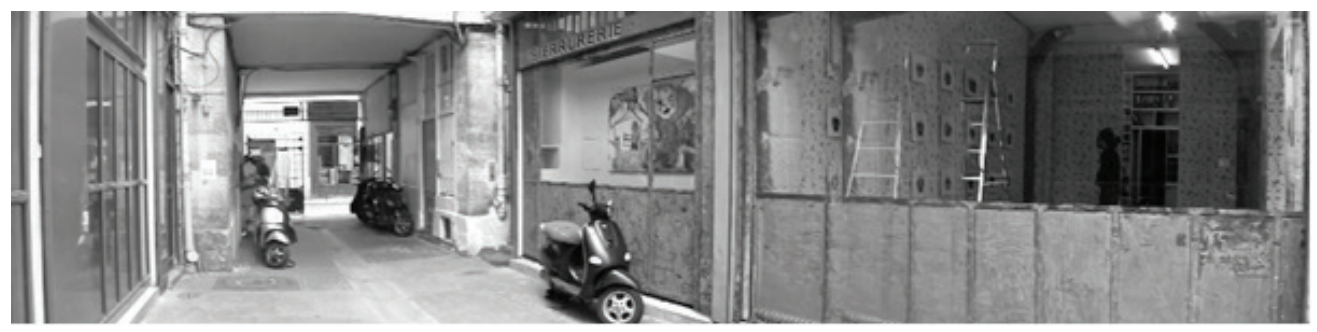

Figure 2 - Montage photographique (capture d'écran) @ Ghislaine Perichet.

Que se passe-t-il entre, ce que je vois et ce que j'entends, ce que j'entendais et voyais, la parenthèse d'espace et de temps à l'origine de Passage des Gravilliers, à l'origine de l'histoire, la fiction?

\section{Le temps d'une expérience}

Quelque chose s'est passé au moment où je posais la caméra sur la chaussée du passage des Gravilliers, au moment où je prenais place face à la galerie Deborah 
Zafman ${ }^{1}$ il y a déjà 3 ans. Une voix féminine appelait, qui rompait le silence de la ruelle désertée et m'interpellait. Une voix dont je tentais de découvrir la provenance, qui me mit littéralement en mouvement, entraînant la caméra dans une lente rotation, mon corps alors « moteur » de l'œil-caméra à la recherche d'un indice susceptible d'éclairer ce qui se passait là, ce que je j'entendais et percevais. J'adaptais ma présence au lieu, à l'événement, le scénario entrain de s'écrire presque à mon insu et pourtant aujourd'hui raconté. Une scène mise en images au rythme de son déroulement, le temps de l'enregistrement, un espace de temps à l'origine d'une expérience, celle de la vidéaste.

\section{Le temps d'une expérience?}

En anglais, reprenant les propos de Richard Shusterman cités en introduction de L'art comme expérience :

comme nom et comme verbe, l'expérience désigne à la fois un événement accompli et un processus ; elle enveloppe à la fois l'instant immédiat et la durée. Elle appartient à la vie et à l'art, et elle est essentielle à l'artiste autant qu'au public. On peut l'interpréter comme une chose qu'une personne engendre par son action, mais aussi comme une chose qu'elle subit ou qui la submerge, comme on peut l'être par le saisissement esthétique².

II y a constamment expérience, rappelait John Dewey, mais pas de manière à composer une expérience. « Nous vivons une expérience lorsque le matériau qui fait l'objet de l'expérience va jusqu'au bout de sa réalisation. [...] Une telle expérience forme un tout ; elle possède en propre des caractéristiques qui l'individualisent et se suffit à elle-même ${ }^{3}$ ". À l'origine de l'expérience vécue au passage des Gravilliers, une voix, un saisissement, un concours de circonstances, le hasard. Une parenthèse d'espace et de temps durant laquelle, actrice tout autant que spectatrice entrainée dans le mouvement de révolution amorcé autour du pied de la caméra, je réalisais l'importance d'un laisser-faire, attentive à ne pas rompre le rythme de la progression circulaire, une vision panoramique bientôt support et cadre du dispositif de la mise en scène à venir, le fil conducteur de la narration.

\section{Forme et processus de formation}

Au déroulé de la scène en perpétuelle révolution s'accordait l'émotion, de celle qui rimerait avec motion. Comme une onde porteuse m'entraînant en une marche régulière, l'émotion est à l'origine du dévoilement du paysage, le fil tendu de l'image en mouvement à partir duquel se déploie le regard, une vision à $360^{\circ}$ dont l'architecture en délimite le périmètre de la sphère, le volume de la scène.

Galerie Deborah Zafman , Le laboratoire des suppositions, 3-5 passage des Gravilliers, 75003 Paris

SHUSTERMAN, R.« Présentation de l'édition française », en introduction de l'ouvrage, p. 19-20.

DEWEY, J. L'art comme expérience. Titre original : Art as experience, 1934), St-Amand, Ed. Gallimard, Coll.

Folio Essais, 2010, p. 80-81. 


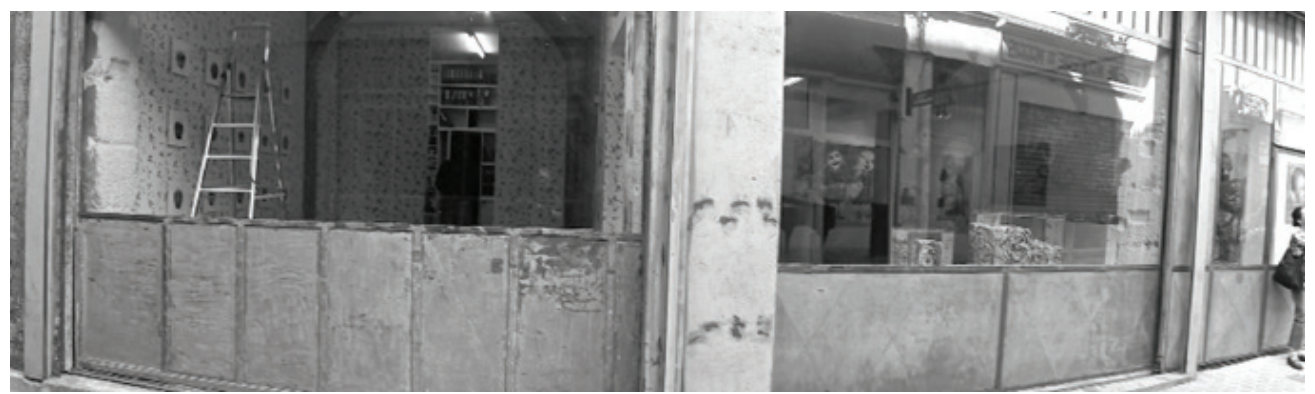

Figure 3 - Montage photographique (capture d'écran) @ Ghislaine Perichet.

L'observateur est pris dans une sphère qui ne se brise jamais, là où il y a des différences qui seront les mouvements et les objets, et dont la surface se conserve close, bien que toutes les portions s'en renouvellent et s'y déplacent. [...] L'observateur n'est d'abord que la condition de cet espace fini : à chaque instant il est cet espace fini ${ }^{4}$.

Visible et mobile mon corps est au nombre des choses, il est l'une d'elles, il est pris dans le tissu du monde et sa cohésion est celle d'une chose. Mais puisqu'il voit et se meut, il tient les choses en cercle autour de soi, elles sont une annexe ou un prolongement de lui-même, elles sont incrustées dans sa chair, elles font partie de sa définition pleine et le monde est fait de l'étoffe même du corps ${ }^{5}$.

Les propos de Paul Valéry, faisant référence aux travaux de Léonard de Vinci, comme ceux de Merleau-Ponty semblent si bien faire écho à l'expérience vécue tant au passage des Gravilliers que dans le contexte du dispositif d'installation de Passage des Gravilliers. Existerait-il un rapport de quasi-équivalence entre le décor, une sphère, cet espace fini, déterminé comme le contexte urbain où se déroulait la scène, l'architecture circonscrivant la scène, et les objets s'y rapportant, les personnages présents au moment du tournage inscrits en cercle autour de soi ? Au contexte de l'enregistrement de l'image vidéo comme au dispositif de sa projection s'apparentent l'espace fini, le tissu du monde et sa cohésion, un lieu dont la vidéaste ou le visiteur, l'observateur est la condition. Mon corps placé au centre de la scène improvisée aura été le point d'orgue autour duquel s'enroulait le paysage urbain, là même où le spectateur prend place dans l'espace de projection, personnage central autour duquel manifestent les voix.

Le travelling de la caméra dévoile l'espace, le volume de la scène, en même temps qu'il le recouvre, la voix de la femme en correspondance avec sa présence à l'écran ou bien perçue hors champ et à même d'intégrer la présence du visiteur, aujourd'hui face à l'écran tout autant qu'immergé dans le contexte sonore. Franchissant le cadre de l'objectif pour faire intrusion sur l'espace de la représentation ou bien s'en extraire, accompagnant ou contrariant le déroulement de l'image pour en dynamiser la composition, tels les acteurs improvisant, l'alchimie des combinaisons hasardeuses engendrées à travers les déplacements des personnages résulte pourtant d'un pur hasard de circonstance. II semble que l'insouciance des uns, combinée à l'émotion d'une autre, sa présence ouverte, ait hier contribué à la réalisation d'un objet aujourd'hui proposé à l'expérimentation du public.

\footnotetext{
4 VALERY, P. « Introduction à la méthode de Léonard de Vinci », in CEuvres, Tome I, Paris, Ed. Gallimard, 1895, p. 1167.

5 MERLEAU-PONTY, M. L’Oeil et l'Esprit, Paris, NRF, Ed. Gallimard (1964), 1983, p. 19-20.
} 
Hier comme aujourd'hui, vidéaste et visiteur se révèlent acteurs bien plus que spectateurs, leur présence respective mobilisée dans le cadre d'un processus dont les facteurs espace, temps et forme, ensemble articulés sous l'effet d'une dynamique, le mouvement panoramique, contribuent à générer le matériau nécessaire à la cristallisation et mise en forme de l'objet Passage des Gravilliers. Unité de lieu, unité de temps, pivot de la scène et moteur du mouvement de la caméra, ma présence associée à chacun des éléments contribuant de près ou de loin à la composition de l'image et donc de la scène, s'inscrivait dans un processus. Le processus catalyseur des relations, que Jean-Louis Le Moigne définit comme « une puissance modélisatrice à l'effet mobilisateur, un ensemble ordonné de changements, pouvant affecté la position dans le temps, dans l'espace, dans

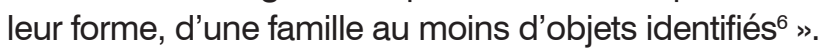

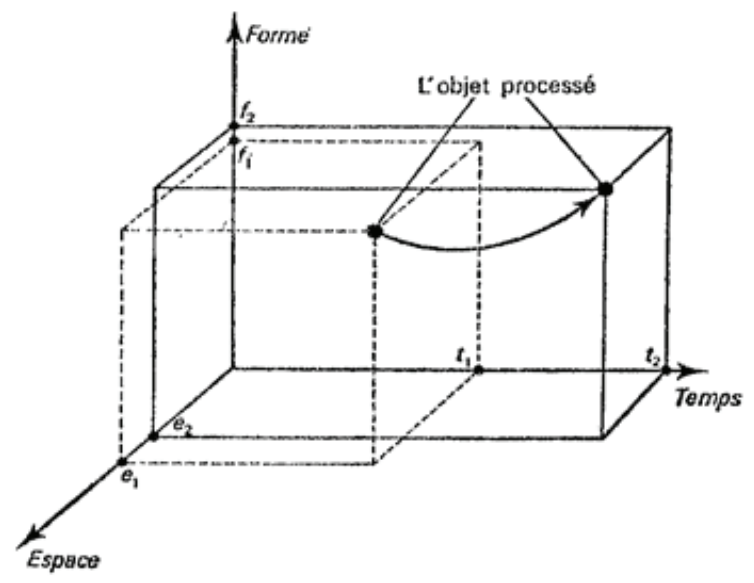

Figure 4 - Le référentiel TEF (Temps, Espace, Forme) permettant de repérer la position des objets soumis à des processus (décomposition triadique des fonctions) ${ }^{7}$.

Encore faut-il y participer à la mise en mouvement des objets et des formes pour qu'advienne alors l'objet, la forme, non pas extrait du contexte d'investigation mais participant pleinement de la réactivation d'un continuum spatio-temporel infini ou, dans le cas de l'expérimentation faite au passage des Gravilliers, au contraire, fini.

Si l'ambiguïté des éléments du paysage est nécessaire à la qualité du paysage, c'est qu'elle nous suggère le passage de l'un à l'autre, et ainsi nous entraîne en un jeu temporel. Cessant d'être des objets, les formes dans l'espace deviennent formes dans le temps - un processus de formation auquel désormais nous participons ${ }^{8}$.

Au moment de la projection, prenant appui sur la structure de l'architecture urbaine, le cadre fixant les limites du champ du regard est pourvoyeur des relations entre les personnages, et le spectateur, alors intégré au dispositif de mise en scène et soumis au processus d'évolution des images et des sons, devenu objet processé et performeur, « opérateur de sa propre expérience » qui, loin d'agir dans l'espace de l'œuvre, (...) est agi par elle ${ }^{9}$. Délimitant le champ de la représentation, le contexte urbain que les

\footnotetext{
LEMOIGNE ,J.-L. La théorie du système général : Théorie de la modélisation, PUF, Paris, p.63.

7 Ibid. 6

8 BERQUES, A. Paysages photographiques, les années quatre-vingt. Mission Datar, Paris, 1990.

9 ROMAN, M. On Stage - La Dimension Scénique de l'Image Vidéo. Paris, Le Gac Press, 2012, p. 156.
} 
personnages traversent tels les acteurs entrant ou sortant des coulisses, les bords du cadre prenant appui sur la structure linéaire des façades accentuent l'effet d'une mise en abyme des cadres au profit d'un cadrage des figures, les voix manifestées alors comme une échappée de la structure. Le dispositif d'installation audiovisuel contribuera à rendre visible et sensible ce cloisonnement des corps alors que les voix s'en extrairont, définissant l'espace de la projection tout en fédérant le hors champ de l'image, visuel et sonore, catalyseur des interférences possibles entre fiction et réalité.

\section{Dispositif et enjeu du corps Entre champ visuel et territoire sonore}

Alors que la ruelle s'appréhende comme l'espace scénique ménagé au profit de la mise en scène impromptue, à l'histoire déroulée en boucle à travers la vision panoramique du passage des Gravilliers s'apparenterait l'historia, l'ordre pictural défini par Alberti comme ordonnateur de la Représentation. Une histoire non pas suspendue, mais un tableau en mouvement, une fiction inscrite dans un continuum spatio-temporel homogène, la traduction d'une réalité où chaque élément constitutif de la scène trouve sa place, chacun interprète son rôle. Unité de lieu, unité de temps, Passage des Gravilliers s'expérimente tel l'espace de temps aménagé au profit d'une présence active, le temps d'une présence tendue, une disponibilité entendue mise en pratique dans la perspective d'expérimenter une situation inopinée pour, plus que spectateur, devenir acteur engagé dans l'aventure.

Hier, pivot à partir duquel s'enroulait l'histoire, la vidéaste fait place aujourd'hui au visiteur invité à prendre place sur scène, s'exposer au déroulement de l'image, à la diffusion du son, son attention retenue entre surface et profondeur, entre champ visuel et territoire sonore. Si l'on peut dire de la couleur, qu'elle nous apparaît là, face à nous, qu'elle est limitée à un endroit donné, délimitant et divisant l'espace en parties qui se déploient en une continuité et une succession réciproques. Le son, par contre, semble avoir une existence propre. Le son nous arrive, nous atteint, nous saisit, flotte à côté de nous, remplit l'espace, le traverse, et se divise en parties qui se suivent dans le temps l'une après l'autre. Alors que le son nous envahit en s'approchant de nous, la couleur demeure à sa place, elle exige du sujet qui la perçoit qu'il se déplace vers elle, qu'il la regarde, qu'il se saisisse d'elle activement. Tous ces aspects n'appartiennent pas à l'objet comme tel, ni même au seul sujet, mais bien à l'expérience perspectiviste du sentir, c'est-à-dire, à la communication entre le Je et le Monde qui est vécue à travers le sentir ${ }^{10}$.

Ainsi l'infime espace de temps durant lequel je réagissais spontanément sans prendre le temps d'interpréter mon geste, l'impulsion du corps mû par l'émotion, hier l'amorce d'une improvisation, serait-il à l'origine de la composition, l'histoire exposée sous la forme d'une installation et aujourd'hui l'objet d'une expérimentation avec le public. Passage des Gravilliers est le carrefour où se joue la combinaison de multiples passages, l'espace construit au profit de nouveaux espaces, entre-deux espaces où se tissent les relations, le lieu de la rencontre avec le public échafaudée entre fiction et réalité : l'image, la scène projetée, et l'espace de la projection, le volume de la représentation.

\footnotetext{
10 STRAUS, E. «Le sentir considéré comme mode de communication » (Unité et pluralité des sens), in Du sens des sens: contribution à l'étude des fondements de la psychologie, Paris, Coll. Krisis, Ed. Jérôme Million, 2000, p. 249.
} 
C'est en prêtant son corps au monde que le peintre change le monde en peinture. Pour comprendre ces transsubstantiations, il faut retrouver le corps opérant et actuel, celui qui n'est pas un morceau d'espace, un faisceau de fonctions, qui est un entrelacs de vision et de mouvement. (...) Mon corps mobile compte au monde visible, en fait partie, et c'est pourquoi je peux le diriger dans le visible. Par ailleurs il est vrai aussi que la vision est suspendue au mouvement. On ne voit que ce qu'on regarde ${ }^{11}$.

N'entend-on que ce que l'on écoute ? II semble que nous entendions mieux, c'està-dire avec acuité, ce que nous connaissons, dans le sens de reconnaître les sons et sonorités auxquels nous aurions déjà été sensibilisés ou confrontés, ceux qui solliciteraient notre attention en faisant écho à un événement, qui raviveraient l'émotion. Comme la femme apparaissait dans mon champ de vision après que sa voix eut détourné mon attention, initié mon mouvement en même temps que l'enregistrement vidéo, c'est au moment où celle-ci quitte la scène, disparaît définitivement du champ de la caméra, sa voix alors tue, que s'annonce la fin de l'événement. Alors qu'hier je suspendais ma ronde silencieuse, le plan-séquence inscrit en boucle perpétue sa course en même temps que se répète la performance. Le temps de l'improvisation s'accorde au temps de la composition, une vision panoramique, l'image circonscrite à l'intérieur du plan-séquence que les bords du cadre de l'objectif, effleurant l'architecture, tendent à infléchir.

Acceptant de participer à l'œuvre, c'est-à-dire d'y être présent et comme, présenté, confronté au dispositif sans y interférer, le public participe de l'histoire en la détournant de son originalité, il en est l'acteur et le garant. Face à l'image panoramique, mais immergé dans l'environnement sonore, le visiteur est à l'origine de la congruence audiovisuelle, il est le corps où s'origine la fiction qui prend forme à l'intérieur du volume de la projection, l'espace dévolu à la représentation. Nous serions là, dirait Georges Didi-Huberman, face à l'écran mais immergé au sein du dispositif audiovisuel, là où

il n'y a pas à choisir entre ce que nous voyons [...] et ce qui nous regarde [...]. Là où, il n'y a qu'à s'inquiéter de l'entre. Là encore où il n'y a qu'à tenter de dialectiser, c'est-à-dire tenter de penser l'oscillation contradictoire dans son mouvement de diastole et de systole [...] à partir de son point central, qui est son point d'inquiétude, de suspens, d'entre-deux. [...] C'est le moment où ce que nous voyons commence juste d'être atteint par ce qui nous regarde [...]. C'est le moment où s'ouvre l'antre creusé par ce qui nous regarde dans ce que nous voyons ${ }^{12}$.

Ce que nous entendons.

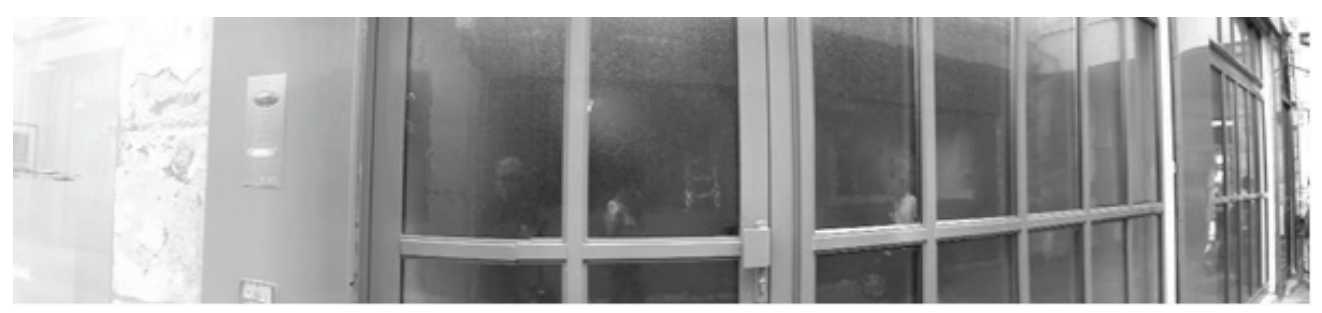

Figure 5 - Montage photographique (capture d'écran) (c) Ghislaine Perichet

\footnotetext{
${ }_{11}$ MERLEAU-PONTY, M. L'Oeil et l'Esprit,Op.cit., 1983, p. 16-17.

12 DIDI-HUBERMAN, G. Ce que nous voyons, ce qui nous regarde. Paris, Ed. Minuit, Collection "Critique", 1992. p. 51-52.
} 


\section{Passage des Gravilliers - Paris, 1er juin 2009}

Passage des Gravilliers, l'histoire hier enregistrée, enroulée le long des surfaces murales à partir de ma présence, moi-même circulant autour de l'axe de la caméra, est aujourd'hui le paysage urbain déroulé à travers le plan de la projection qui continue sa ronde infiniment. Comme hier, moi-même au centre de l'arène, happée par l'événement, le public est aujourd'hui confronté à la projection vidéo, immergé dans le contexte sonore enregistré simultanément à l'image, sa présence sollicitée au sein d'un complexe audiovisuel, son attention retenue entre champ visuel et territoire sonore, entre le flux ininterrompu du défilement de l'image panoramique et l'interférence des voix à même d'en rompre la monotonie.

Comme hier, au passage des Gravilliers, le temps de mon expérience éprouvée, le visiteur est aujourd'hui présent à l'intérieur de l'espace d'exposition. II est le spectateur privilégié, le centre du dispositif soumis à l'épreuve de l'image et des sons, à même de reconsidérer l'histoire, et peut-être la faire sienne. Passage des Gravilliers, pour reprendre les mots d'Alexandre Bohn décrivant l'œuvre du plasticien Krijn De Koning, n'est définitivement pas

un objet à admirer en soi, mais un dispositif expérimental dont la pratique doit permettre chez le visiteur un renouvellement de la perception d'une réalité préexistante (un lieu, un contexte, son propre corps, son propre statut) et un accès à un discernement plus aigu ${ }^{13}$.

Comme j'explorais l'espace dévolu à la représentation, la caméra explorait le temps. Le temps de l'avènement d'une expérience, pendant que le technicien indifférent à la scène continue d'oeuvrer derrière la vitrine de la galerie, alors que l'homme à la chemise blanche traverse d'un pas décidé le champ de la caméra et que la jeune femme, à peine aperçue en fond de ruelle, déjà disparue. Chacun a sa place. Chacun à sa place.

\section{Références}

BERQUES, Augustin. Paysages photographiques, les années quatre-vingt. Mission Datar, Paris, Hazan, 1990.

von BERTALANFFY, Ludwig. Théorie générale des systèmes (traduit par Jean Benoist Chabrol), Paris, Dunod, 1973.

BOHN, Alexandre. « Krijn de Koning », in revue Art Press, FRAC Lorraine, Metz, Juillet 2001.

DEWEY, John. Art as experience, (1934), L'Art comme expérience, CEuvres philosophiques III, (traduction de l'anglais par Jean-Pierre Cometti et alii), Éditions Farrago/Université de Pau, 2005 et Gallimard, 2010.

DIDI-HUBERMAN, Georges. Ce que nous voyons, ce qui nous regarde, Paris, Ed. Minuit, Collection "Critique", 1992.

${ }_{13}$ BOHN, A. « Krijn de Koning », in Art Press, FRAC Lorraine, Metz, Juillet 2001. 
LEMOIGNE, Jean-Louis. La théorie du système général - Théorie de la modélisation, Collection Les Classiques du réseau intelligent de la complexité, Paris, PUF, 1977.

MERLEAU-PONTY, Maurice. L’Oeil et I’Esprit, Paris, NRF, Gallimard (1964), 1983.

ROMAN, Mathilde. On Stage - La Dimension Scénique de I'Image Vidéo, Paris, Le Gac Press, 2012.

STRAUS, Erwin. «Le sentir considéré comme mode de communication » (Unité et pluralité des sens), in Du sens des sens: contribution à l'étude des fondements de la psychologie, Paris, Coll. Krisis, Ed. Jérôme Million, 2000.

VALÉRY, Paul. Introduction à la méthode de Léonard de Vinci. in CEuvres, Tome I, Paris, Gallimard, 1895. 